\title{
INFLATIONARY SOLUTIONS OF BIANCHI TYPE I STRING MODELS WITH BULK VISCOUS FLUID IN BIMETRIC THEORY OF GRAVITATION
}

\author{
Vijay Lepse \\ Department of Mathematics, Science College Pauni-441910
}

\begin{abstract}
In this paper, we presented the solutions of inflationary solutions of bianchi type i string models with bulk viscous fluid in bimetric theory of gravitation. The bulk viscosity leads inflationary like solutions in the mode with cosmetic string and dominated the nature of Hubbell parameter, the expansion $\theta$ and shar $\sigma$ in the evaluation of the universe Keywords: Cosmology, Viscosity
\end{abstract}

\section{Introduction}

Bulk viscous fluid has major contribution in the evaluation of the universe at early stage as well as at later stage. On large scale distribution of galaxies in the universe shows that the matter distribution is satisfactorily described by the perfect fluid. However, when neutrino decoupling occurs, then the matter behaves like viscous fluid in early stage of the universe. The researchers like Misner (1967, 1968) suggested that strong dissipation due to the neutrino viscosity may considerably reduce the anisotropy of the black-body radiation. Weinberg (1972) has described that the viscosity mechanism in cosmology that can explain the anomalously high entropy per baryon in the present universe. Belinskii and Khalatnikow (1975) have suggested the nature of cosmological solutions for the homogeneous Bianchi type I models by taking into account the dissipative process due to viscosity. Chimento et al. (1997) have investigated the cosmological models with bulk viscosity and studied their nature. Also there are many researchers like Santos et al. (1985), Banerjee et al. (1985), Huang (1990), Desikan (1997), Krori et al. (2000),Bali et al. (2002), Singh et al. (2008), Xu Dou et al. (2011), Tiwari et al. (2011), ), Verma (2011), Gilberto et al. (2012), Sharma (2012), Zeyauddian et al. (2013), Singh et al. (2013), Rao et al. (2013) and Benaum (2014) who have evaluated the models of the universe with bulk viscous fluid and discussed the effect of bulk viscosity on the evaluation of universe.

\section{Metric and Rosen's Field Equations}

We consider the Bianchi Type I metric

$d s^{2}=-d t^{2}+A^{2} d x^{2}+B^{2} d y^{2}+C^{2} d z^{2}$

where $A, B$ and $C$ are the functions of $t$ only.

The flat metric corresponding to metric (5.2.1) is

$d \eta^{2}=-d t^{2}+d x^{2}+d y^{2}+d z^{2}$

The energy momentum tensor $T_{i}{ }^{j}$ of the source of bulk viscous fluid with string is given by

$$
T_{i}^{j}=\in v_{i} v^{j}-\lambda x_{i} x^{j}-\xi v_{\mid l}^{l}\left(g_{i}^{j}+v_{i} v^{j}\right)
$$

with

$$
v_{i} v^{j}=-x_{i} x^{j}=-1
$$

and

$$
v^{i} x_{i}=0
$$

Here $\epsilon$ is the rest energy density and $\epsilon=\epsilon_{p}+\lambda$ where $\epsilon_{p}$ and $\lambda$ denote the particle density (particles loaded on the string) and the string tension density respectively, $x^{i}$ is the direction of string, $v^{i}$ is the four velocity vector, $\xi$ is the coefficient of bulk viscosity and $\theta$ is the scalar expansion which is given by 
$\theta=v_{\mid l}^{l}$

Thus, the Rosen's field equations are,

$-\frac{\ddot{A}}{A}+\frac{\ddot{B}}{B}+\frac{\ddot{C}}{C}+\frac{\dot{A}^{2}}{A^{2}}-\frac{\dot{B}^{2}}{B^{2}}-\frac{\dot{C}^{2}}{C^{2}}=16 \pi A B C(\lambda+\xi \theta)$

$\frac{\ddot{A}}{A}-\frac{\ddot{B}}{B}+\frac{\ddot{C}}{C}-\frac{\dot{A}^{2}}{A^{2}}+\frac{\dot{B}^{2}}{B^{2}}-\frac{\dot{C}^{2}}{C^{2}}=16 \pi A B C \xi \theta$

$\frac{\ddot{A}}{A}+\frac{\ddot{B}}{B}-\frac{\ddot{C}}{C}-\frac{\dot{A}^{2}}{A^{2}}-\frac{\dot{B}^{2}}{B^{2}}+\frac{\dot{C}^{2}}{C^{2}}=16 \pi A B C \xi \theta$

$\frac{\ddot{A}}{A}+\frac{\ddot{B}}{B}+\frac{\ddot{C}}{C}-\frac{\dot{A}^{2}}{A^{2}}-\frac{\dot{B}^{2}}{B^{2}}-\frac{\dot{C}^{2}}{C^{2}}=16 \pi A B C \in$

From differential equations, we write

$\frac{d}{d t}\left(\frac{\dot{B}}{B}\right)=\frac{d}{d t}\left(\frac{\dot{C}}{C}\right)$

which on integrating, we get

$B=a_{1} C$

where $a_{1}$ is constant of integration and choosing the constant of integration $a_{1}=1$, so that

$B=C$

Using equation (2.11), the differential equations can be written as

$-\left(\frac{\ddot{A}}{A}-\frac{\dot{A}^{2}}{A^{2}}\right)+2\left(\frac{\ddot{B}}{B}-\frac{\dot{B}^{2}}{B^{2}}\right)=16 \pi A B^{2}(\lambda+\xi \theta)$

$\left(\frac{\ddot{A}}{A}-\frac{\dot{A}^{2}}{A^{2}}\right)=16 \pi A B^{2} \xi \theta$

$\left(\frac{\ddot{A}}{A}-\frac{\dot{A}^{2}}{A^{2}}\right)+2\left(\frac{\ddot{B}}{B}-\frac{\dot{B}^{2}}{B^{2}}\right)=16 \pi A B^{2} \in$

On subtracting equations (2.14) and (2.15), we get

$-2\left(\frac{\ddot{A}}{A}-\frac{\dot{A}^{2}}{A^{2}}\right)+2\left(\frac{\ddot{B}}{B}-\frac{\dot{B}^{2}}{B^{2}}\right)=16 \pi A B^{2} \lambda$

Adding equations (2.15) and (2.16), we have

$-\left(\frac{\ddot{A}}{A}-\frac{\dot{A}^{2}}{A^{2}}\right)+4\left(\frac{\ddot{B}}{B}-\frac{\dot{B}^{2}}{B^{2}}\right)=16 \pi A B^{2}(\lambda+\epsilon)$

The conservation law for the energy-momentum tensor, yield

$\dot{\epsilon}=(-2 \in+\lambda) \frac{2 \dot{B}}{B}+\xi \theta \frac{4 \dot{B}}{B}$

We are going to solve these two differential equations (2.17) and (2.18) in Five unknowns $A, B, \lambda, \in$ and $\xi$. Here $\theta$ is a known quantity with the function of $A$ and $B$. In order to have solution, we assume 
three extra conditions. First we assume the relation between $A$ and $B$ (the scalar expansion $\theta$ is proportional to shear $\sigma$ assumed by Saha (2005)) as

$A=B^{2}$

On differentiating the above equation, we get

$$
\left(\frac{\ddot{A}}{A}-\frac{\dot{A}^{2}}{A^{2}}\right)=2\left(\frac{\ddot{B}}{B}-\frac{\dot{B}^{2}}{B^{2}}\right)
$$

After solving the above equations, we have

$$
\frac{\ddot{B}}{B}-\frac{\dot{B}^{2}}{B^{2}}=8 \pi B^{4}(\lambda+\in)
$$

Assuming the second condition $\xi \theta=\mu$ (constant), i.e., the coefficient of bulk viscosity $\xi$ varies with the scalar expansion $\theta$, since the viscosity, being dissipative phenomenon, is believed to affect the expansion of the universe. Originally, this condition is assumed by Santos et al. (1985) and then many other researchers like Saha (2005) and Bali et al. (2007), etc.

The rest energy density $\in$ and the string tension density $\lambda$ for the composite matter of viscous fluid and dark energy can be taken together as

$$
\in=\epsilon_{V F}+\epsilon_{D E}, \quad \lambda=\lambda_{V F}+\lambda_{D E}
$$

and the energy momentum tensor equation (5.2.3) can be decomposed as

$T_{i}^{j}=\left(\epsilon_{V F}+\epsilon_{D E}\right) v_{i} v^{j}-\left(\lambda_{V F}+\lambda_{D E}\right) x_{i} x^{j}-\mu\left(g_{i}^{j}+v_{i} v^{j}\right)$

in which $\in_{D E}$ is the component of energy density corresponding to dark energy and $\lambda_{D E}$ is the component of string tension density corresponding to dark energy. The term $\in_{V F}$ is the rest energy density and $\lambda_{V F}$ is the string tension density corresponding to the viscous fluid.

\section{Model with Viscous Fluid}

The viscous fluid obeys the equation of state

$$
\lambda_{V F}=\gamma \in_{V F}, \quad 0 \leq \gamma \leq 1
$$

Here $\gamma=0$ (Dust Universe), $\gamma=\frac{1}{3}$ (Radiation Universe), $\gamma=\left(\frac{1}{3}, 1\right)$ (Hard Universe) and $\gamma=1$ (Zel'dovich Universe or stiff matter).

In view of this energy density $\in_{V F}$ and string tension density $\lambda_{V F}$, from the equation (3.1), the differential equation becomes

$$
\begin{aligned}
& \frac{\ddot{B}}{B}-\frac{\dot{B}^{2}}{B^{2}}=8 \pi B^{4}\left(\lambda_{V F}+\epsilon_{V F}\right) \\
& \dot{\epsilon}_{V F}+\dot{\epsilon}_{D E}=\left(-2\left(\epsilon_{V F}+\epsilon_{D E}\right)+\left(\lambda_{V F}+\lambda_{D E}\right)\right) \frac{2 \dot{B}}{B}+\mu \frac{4 \dot{B}}{B}
\end{aligned}
$$

In the evaluation of energy density $\in$ and string tension density $\lambda$, in case of bulk viscous fluid and dark energy, we bifurcate the conservation law equation (5.3.2) as 


$$
\dot{\epsilon}_{V F}=\left(-2 \epsilon_{V F}+\lambda_{V F}\right) \frac{2 \dot{B}}{B}
$$

With the help of equation of state (3.1), we write equation (3.3) as,

$$
\begin{aligned}
& \dot{\epsilon}_{V F}=\left(-2 \epsilon_{V F}+\gamma \in_{V F}\right) \frac{2 \dot{B}}{B} \\
& \Rightarrow \dot{\epsilon}_{V F}=2(\gamma-2) \epsilon_{V F} \frac{\dot{B}}{B} \\
& \Rightarrow \frac{\dot{\epsilon}_{V F}}{\epsilon_{V F}}=2(\gamma-2) \frac{\dot{B}}{B}
\end{aligned}
$$

On integrating, we get

$$
\epsilon_{V F}=\alpha B^{2(\gamma-2)}
$$

where $\alpha$ is an integrating constant.

Using the above value of energy density $\in_{V F}$, we get string tension density $\lambda_{V F}$ as

$$
\lambda_{V F}=\gamma \alpha B^{2(\gamma-2)}
$$

Thus, the energy density $\in_{V F}$ and string tension density $\lambda_{V F}$ corresponding to the viscous fluid model are given by

$$
\epsilon_{V F}=\alpha B^{2(\gamma-2)} \quad \text { and } \quad \lambda_{V F}=\gamma \alpha B^{2(\gamma-2)}
$$

OR

$$
\frac{\ddot{B}}{B}-\frac{\dot{B}^{2}}{B^{2}}=8 \pi \alpha(1+\gamma) B^{2 \gamma}
$$

we are going to solve the integral equation (3.5) and by straight forward calculations, we arrived at the solution

$$
B=\left(b \sinh \left(c_{2}-\sqrt{c_{1}} \gamma t\right)\right)^{-1 / \gamma}
$$

in which $c_{1}$ and $c_{2}$ are constants of integration and $b=\left(8 \pi \alpha(1+\gamma) / \gamma c_{1}\right)^{1 / 2}=$ constant Using the equation(3.6), we write the values of $A$ and $C$ as

$$
\begin{aligned}
& A=\left(b \sinh \left(c_{2}-\sqrt{c_{1}} \gamma t\right)\right)^{-2 / \gamma} \\
& C=\left(b \sinh \left(c_{2}-\sqrt{c_{1}} \gamma t\right)\right)^{-1 / \gamma}
\end{aligned}
$$

The volume $V$ is

$$
V=\left(b \sinh \left(c_{2}-\sqrt{c_{1}} \gamma t\right)\right)^{-4 / \gamma}
$$

Here it is to be noted that the equation of state parameter $\gamma$ is non-zero i.e., $\gamma \neq 0$, since the scale factors $A, B, C$ and the volume $V$ do not exist at $\gamma=0$. For $\gamma \neq 0$, the required metric corresponding to viscous fluid is 


$$
\begin{aligned}
d s^{2}=-d t^{2} & +\left(b \sinh \left(c_{2}-\sqrt{c_{1}} \gamma t\right)\right)^{-4 / \gamma} d x^{2} \\
& +\left(b \sinh \left(c_{2}-\sqrt{c_{1}} \gamma t\right)\right)^{-2 / \gamma}\left(d y^{2}+d z^{2}\right)
\end{aligned}
$$

In this viscous fluid model for $\gamma \neq 0$, the physical parameters like scalar expansion $\theta$, the coefficient of bulk viscosity $\xi$, anisotropic parameter $A$, the shear scalar $\sigma$, and the deceleration parameter $q$ are

$$
\begin{aligned}
& \theta=4 \operatorname{coth}\left(1-\frac{t}{2}\right), \quad \xi=\frac{\mu}{4 \operatorname{coth}\left(1-\frac{t}{2}\right)}, \quad A=\frac{1}{8} \\
& \sigma^{2}=\frac{1}{3} \operatorname{coth}^{2}\left(1-\frac{t}{2}\right), \quad q=-\frac{3}{8} \operatorname{sech}^{2}\left(1-\frac{t}{2}\right)-1
\end{aligned}
$$

The nature of scalar expansion $\theta$ and shear $\sigma$ have been shown in similar in nature to that of volume $V$ and Hubble parameter $H$ and they do not contribute any new behavior geometrically and physically. The bulk viscosity coefficient $\xi$ has the direct connection with the Hubble parameter $H$ (which has the scaling relation with the red shift), the expansion $\theta$ and the shear $\sigma$ in the evaluation of the universe and it dominate $H, \theta$ and $\sigma$. For small effect of $\xi$, the rate of expansion and shear both are very high and they slowing down as $\xi$ increases, and the rate of expansion stopped and shear vanish, for high range of $\xi$.

\section{Conclusion}

Bianchi type I models with the assumptions of bulk viscous fluid and dark energy have been deduced by solving the Rosen's field equations in Bimetric theory of gravitation and observed that the bulk viscosity leads inflationary like solutions in the evaluation of the universe. The string viscous fluid models for $0<\gamma \leq 1$, starts evolving with non-zero volume, non-zero energy density, string tension density and particle density and goes over to vacuum (matter died) at $t=2$ and further the matter suddenly appeared in the model whose energy density, particle density and string phases goes on increasing. The bulk viscosity coefficient $\xi$ has the direct connection with the Hubble parameter $H$ (which has the scaling relation with red shift), with the expansion $\theta$ and with the shear $\sigma$ in the evaluation of the universe and it dominates $H, \theta$ and $\sigma$. For small effect of $\xi$, the rate of expansion and shear both are very high and they slowing down as $\xi$ increases and the rate of expansion stopped and shear vanishes, for high range of $\xi$. The deceleration parameter $q$ is negative shows the model is acceleratingly expanding. It is anisotropize and has shear. We have deduced the solutions of Rosen's field equations for Bianchi type I space-time in the presence of string bulk viscous fluid and dark energy quintessence and Chaplygin gas. The bulk viscosity $\xi$ leads inflationary like solutions in the models with cosmic strings and dominated the nature of Hubble parameter $H$, the expansion $\theta$ and the shear $\sigma$ in the evaluation of the universe. Five models have been investigated namely, Non-dust string viscous fluid model, Dust string viscous fluid model, Quintessence dark energy model with viscosity for $-1<\omega_{q}<1$ and for $\omega_{q}=-1$ and chaplygin gas dark energy. It is noticed that the geometry of quintessence dark energy model, for $-1<\omega_{q}<0$ is coincide with the geometry of non-dust string fluid model for $0<\gamma \leq 1$. The dust string viscous fluid model and Chaplygin gas dark energy model yield same geometry except some physical behavior. Other geometrical and physical aspects of the models are studied. 


\section{Refference}

[1] S.Perlmutter et al.Nature, vol. 391, pp. 51(1998). [2] A.G. Riess et al., Astron. J., vol. 116, pp.1009 (1998).

[3] S.Perlmutter et al., Astrophys. J.,vol. 517, pp.565 (1999).

[4] R.A. Knop et al., Astrophys. J.,vol. 598, pp.102 (2003).

[5] A.G. Riess et al., Astrophys. J.,vol. 607,pp. 665 (2004).

[6] C.L. Bennett et al., Astron. Astrophys.Suppl.

Ser., vol. 148, pp. 1 (2003).

[7] D.N. Spergel et al., Astron. Astrophys. Suppl.

Ser.,vol. 148, pp. 175 (2003).

[8] M.Tegmark et al. (SDSS Collaboration), Phys.

Rev.D,vol. 69, pp.103501(2004a).

[9] M.Tegmark et al. (SDSS Collaboration),

Astrophys. J.,vol. 606, pp. 702 (2004b).

[10] U.Seljak et al.,Phys. Rev.D, vol.71, pp.103515 (2005).

[11] J.K.Adelman-McCarthy et al. (SDSS

Collaboration) (2005).

[12] K.Abazajian et al. (SDSS Collaboration), (2003).

[13] K.Abazajian et al. (SDSS Collaboration) (2004).

[14] K. Abazajianet al. (SDSS Collaboration): (2004).

[15] B. Ratra, P.J.E. Peebles, Phys. Rev. D, vol. 37, pp. 3406 (1988).

[16] C.Wetterich, Nucl. Phys.,vol.B302, pp. 668 (1988).

[17] A.R Liddle, R.J Scherrer, Phys. Rev.D,vol. 59, pp. 023509 (1999).

[18] I.Zlatev,L.M. Wang, P.J. Steinhardt, Phys.

Rev. Lett., vol. 82, pp. 896 (1999).

[19] R.R. Caldwell, Phys. Lett. B,vol. 545, pp. 23

(2002).

[20] R.R.Caldwell,M. Kamionkowski, N.N.

Weinberg, Phys. Rev. Lett., vol. 91,

pp.071301(2003).

[21] S. Nojiri,S.D. Odintsov, Phys. Lett. B,vol. 562, pp.147 (2003).

[22] V.K. Onemli, R.P. Woodard, Phys. Rev. D, vol. 70, pp. 107301 (2004).

[23] M. R. Setare, J. Sadeghi, A.R. Amani, Phys.

Lett. B,vol. 666, pp. 288 (2008).

[24] B. Feng,X.L. Wang, X.M. Zhang, Phys. Lett.

B,vol. 607, pp. 35(2005).

[25] Z.K. Guo et al., Phys. Lett. B,vol. 608, pp.177 (2005).

[26] M.-Z. Li,B. Feng,X.-M. Zhang, J. Cosmol. Astropart. Phys.,vol. 002, pp. 0512 (2005).

[27] B. Feng,M. Li, Y.-S. Piao, X. Zhang, Phys.

Lett. B,vol. 634, pp.101 (2006).

[28] W. Zhao, Y. Zhang, Phys. Rev. D, vol. 73, pp. 123509 (2006).
[29] M.R.Setare, J.Sadeghi, A.R. Amani, Phys. Lett. B,vol. 660, pp.299 (2008).

[30] J. Sadeghi, M.R. Setare, A. Banijamali, F. Milani, Phys. Lett. B,vol.662, pp.92 (2008).

[31] M.R. Setare, E.N. Saridakis, Phys. Lett. B,vol. 668, pp.177 (2008).

[32] M. R. Setare, E.N. Saridakis, arXiv:0807.3807 [hep-th].

[33] M.R. Setare, E.N. Saridakis, J. Cosmol

Astropart. Phys., vol. 0809, pp. 026 (2008).

[34] A.Y. Kamenshchik, U. Moschella, V.

Pasquier, Phys. Lett. B, vol. 511, pp. 265 (2001).

[35] M.R. Setare, Eur. Phys. J., vol. C52, pp. 689 (2007).

[36] M.R. Setare, Int. J. Mod. Phys.D,vol. 18, pp. 419 (2009).

[37] J. Kujat et al., Astrophys. J.,vol. 572, pp. 1 (2002).

[38] M. Bartelmann et al., New Astron. Rev.,vol. 49, pp. 199 (2005).

[39] R.Jimenez, New Astron. Rev., vol. 47, pp. 761 (2003).

[40] A.Das et al., Phys. Rev. D.,vol. 72, pp. 043528 (2005).

[41] M.S.Turner,M. White, Phys. Rev. D., vol. 56, pp. R4439 (1997).

[42] R.R.Caldwell et al., Phys. Rev. Lett., vol. 80, pp. 1582 (1998).

[43] A.R.Liddle, R.J. Scherrer, Phys. Rev. D, vol [44] N.Rosen, Plenum Press, London, pp. 273-94 (1977).

[45] T.M. Karade, Indian J. Pure-appl. Math, vol. 11(9), pp. 1202 (1980).

[46] M.Isrelit, Gen. Relativ. Gravit., vol. 13(7), pp. 681 (1981).

[47] D.R.K. Reddy, N. Venkateswara Rao,

Astrophys. Space Sci., vol. 257, pp. 293 (1998).

[48] R. Bali, Anjali, Astrophys Space Sci.,vol. 302, pp. 201 (2006).

[49] R.Bali,S. Dave, Astrophys. Space Sci., vol.

288, pp. 503 (2003).

[50] R.Bali, R.D. Upadhaya, Astrophys. Space Sci. vol. 283, pp. 97 (2003).

[51] R. Bali, D. Singh, Astrophys. Space Sci., vol. 300, pp. 387 (2005).

[52] R. Bali, U.K. Pareek, Astrophys. Space Sci., vol. 312, pp. 305 (2007).

[53] S.D. Katore, R.S. Rane, Pramana J. Phys., vol. 67(2), pp. 237 (2006).

[54] G.S. Khadekar, S.D. Tade, Astrophys. Space Sci. (1998) DOI 10.1007\S10509 - 007- 9410-2.T. [55] M.S. Borkar, S.S. Charjan, IJAM, vol. 22(3), pp. 445, (2009).

[57] M.S. Borkar, S.S. Charjan, J. Ind. Acad. Math, vol. 32(1), (2010).

[58] M.S. Borkar, S.S. Charjan, An Int. J. AAM, vol. 5(1), pp. 96109 (2010). 
[59] M.S. Borkar, S.S. Charjan, An Int. J. AAM , vol. 5(2), pp. 1660 (2010)
[60] N.P. Gaikwad et al.: Chin. Phys. Lett.,vol.28(8), pp. 089803 (2011) 